\title{
The 2016 update to NICE CG95 guideline for the investigation of new onset stable chest pain: more innovation, but at a cost?
}

\author{
Authors: Khaled Alfakih, ${ }^{A}$ John P Greenwood ${ }^{B}$ and Sven Plein ${ }^{C}$
}

The National Institute for Health and Care Excellence (NICE) published an update on its guideline on chest pain of recent onset in 2016. The new guideline makes three key changes to the $\mathbf{2 0 1 0}$ version. NICE recommend that the previously proposed pre-test probability risk score should no longer be used. They also recommend that a calcium score of zero should no longer be used to rule out coronary artery disease in patients with low pre-test probability. However, the most radical change is that NICE now recommend that all patients with new onset chest pain should be investigated with a computerised tomography coronary angiogram as a first-ine investigation. We discuss the recent trial evidence that has informed the NICE update and consider the impact of the new guidelines.

KEYWORDS: Coronary artery disease, CT coronary angiography, guidelines, NICE, stable chest pain

The National Institute for Health and Care Excellence (NICE) has just published an update of its guideline on recent onset chest pain (CG95). The original guideline was published in 2010 and was a radical departure from established clinical practice. ${ }^{1}$ It recommended that the exercise electrocardiogram (ECG) was no longer used to investigate patients with recent onset stable chest pain. Instead NICE recommended that the choice of investigation was determined by the pre-test probability (PTP) risk score (RS), which - based on the Duke clinical score - was calculated using age, gender, typicality of chest pain and the presence of cardiovascular risk factors. ${ }^{2}$ The guidance recommended that patients with an RS $<10 \%$ should not undergo cardiac investigation and patients with an RS of $10-29 \%$ were to undergo computerised tomography

Authors: ${ }^{\text {A }}$ consultant cardiologist, Department of Cardiology, University Hospital Lewisham, London, UK; ${ }^{\text {B }}$ rofessor of cardiology, Multidisciplinary Cardiovascular Research Centre \& Leeds Institute of Cardiovascular and Metabolic Medicine, University of Leeds, Leeds, UK; ' professor of cardiovascular imaging, Multidisciplinary Cardiovascular Research Centre \& Leeds Institute of Cardiovascular and Metabolic Medicine, University of Leeds, Leeds, UK
(CT) calcium scoring. A zero calcium score was taken as confirmation of very low likelihood of coronary artery disease (CAD) and no further investigation was needed. If the calcium score was above zero, a CT coronary angiogram (CTCA) was recommended. Patients with an intermediate RS of 30-60\% were to have a functional imaging test, such as myocardial perfusion scintigraphy (MPS), stress echocardiography or stress cardiovascular magnetic resonance imaging. Patients with high RS of 61-90\% were recommended to directly undergo invasive coronary angiography (ICA) and patients with an RS $>90 \%$ were assumed to have CAD without further testing.

The NICE guideline update (2016) makes three key changes to the 2010 version. ${ }^{3}$ The first is the recommendation for a clinical assessment of the likelihood of CAD, based on the typicality of the chest pain into typical, atypical or non-cardiac, instead of the previous PTP RS. The second change in the guideline is that a zero calcium score is no longer used to rule out CAD in patients with low PTP. Thirdly, and most radically, NICE now recommends that all patients with new onset chest pain with atypical or typical anginal features, as well as those with noncardiac chest pain and an abnormal resting ECG, should first be investigated with CTCA using a 64-slice (or above) CT scanner. Functional imaging tests are now reserved for the assessment of patients with chest pain symptoms who are known to have CAD and for patients where the CTCA has been non-diagnostic or has shown CAD of uncertain significance.

Some of the changes made to the guidelines by NICE put them at odds with international recommendations. For example, the 2013 European Society of Cardiology (ESC) guideline on stable chest pain recommends the use of a PTP RS that is calculated using age, gender and typicality of chest pain, but not cardiovascular risk factors. ${ }^{4}$ This RS is based on an updated Diamond-Forrester method, which adjusts the likelihood of CAD for a more contemporary population. ${ }^{5}$ The ESC recommends that patients with an intermediate RS (15-85\%) have a functional imaging test and, if there is limited availability, exercise ECG is recommended as an alternative in patients with RS $15-65 \%$ and CTCA for patients with RS $15-50 \%$.

The 2012 guidelines from the American cardiology societies on stable chest pain recommend clinical evaluation of the PTP of CAD. ${ }^{6}$ Patients able to exercise, with interpretable resting ECGs and a low to intermediate likelihood of CAD are recommended to have an exercise ECG. Patients with 
uninterpretable ECG and patients with intermediate to high likelihood of CAD are recommended to have functional imaging tests. Patients with low to intermediate PTP, who are unable to exercise may also undergo CTCA as an alternative to exercise stress testing.

\section{The 2016 update to NICE CG95 guideline}

In the latest guidance, NICE has parted from its PTP model, the rationale being that it overestimated risk in current rapid access chest pain clinic populations, something that NICE anticipated in their 2010 guideline and was subsequently confirmed in registries. ${ }^{7,8}$ The NICE PTP RS model overestimates risk because it was based on cohorts of patients undergoing ICA in the USA in the 1970s who had a much higher prevalence of CAD than current rapid access chest pain clinic populations. There was an expectation that NICE may adopt the ESC RS, which is based on a contemporary population and has been externally validated, and shown to be a good predictor of risk. ${ }^{8,9}$

However, the most striking change in the new NICE guideline is the expansion of the use of CTCA to all patients with new onset of chest pain. NICE no longer recommends coronary artery calcium scoring followed by CTCA if the calcium score is above zero because of case reports of significant coronary stenoses in patients with a zero calcium score. Another reason is that the radiation dose from CTCA on high specification CT scanners is now as low as the radiation dose for the calcium score itself (less than $1 \mathrm{mSv}$ ). More controversially, NICE expanded the recommendation for CTCA as first line to patients with intermediate and high likelihood of CAD based on their cost-effectiveness analysis suggesting that this would be a lower cost strategy. While recent clinical trials, such as PROMISE, demonstrated that patients investigated with CTCA and functional tests had similar clinical outcomes, ${ }^{10}$ one has to remember that this trial was in a low-intermediate disease prevalence population with only approximately $11 \%$ having CAD. Although the SCOT-HEART trial had a higher prevalence of CAD and demonstrated that the use of CTCA in patients with chest pain improves the diagnosis when added to standard of care, the standard of care was the exercise ECG and not functional imaging tests. ${ }^{11}$ In fact, there are no published data demonstrating the diagnostic accuracy or cost effectiveness of CTCA in patients with chest pain and higher likelihood of CAD, making the NICE recommendations for CTCA in this population somewhat surprising. Interestingly, however, there are UK data demonstrating higher utilisation rates of the costly ICA following a CTCA strategy. ${ }^{12}$

\section{The radiation dose}

The practical implementation of the new guidelines will meet many challenges. The recommendations are in part based on the assumption that the radiation dose of CTCA is in the order of $1-2 \mathrm{mSv}$, which is achievable in most patients with the latest generation CT scanners. However, most UK hospitals don't have these and instead use 64-slice CT scanners that can perform a CTCA with a radiation dose of of 3-5 $\mathrm{mSv}$, providing prospective gating is used, which requires a heart rate of $60 \mathrm{bpm}$; otherwise, CTCA is performed with retrospective gating, which allows for a heart rate up to $70 \mathrm{bpm}$, but with radiation doses of $10-15 \mathrm{mSv}$, a similar radiation dose to MPS. ${ }^{13}$ In PROSPECT, a trial comparing CTCA and MPS in patients with intermediate risk chest pain, the total radiation dose was high in both arms ( 24 versus $29 \mathrm{mSv}$ ) and no difference was found in the rates of ICA between the two strategies. ${ }^{14}$ To implement the NICE guidelines without increasing the radiation burden on the population, the NHS will need to make a significant investment in high-specification CT scanners and/or carefully consider the choice of the follow on functional imaging test, based on the patient's age, sex and their cumulative radiation dose, from other radiation-based investigations. A further major challenge, if the NICE recommendations are to be adopted, will be to identify and train the increased requirement for radiographers and consultants to perform and report the additional CTCAs.

\section{Conclusions}

CTCA is an excellent rule-out test for CAD when used in the appropriate disease prevalence population. Despite the recent NICE update to CG95, there is little or no clinical or health economic data to support the use of CTCA over other noninvasive imaging tests in patients at intermediate-high risk of CAD. Furthermore, it appears that the impact on downstream testing has not been considered, nor has the potential for high cumulative radiation dose exposure from multiple serial investigations, based on current UK CT scanners. Whether UK hospitals could fundamentally reconfigure their chest pain investigation pathways, based on current finances and staffing levels, also remains to be seen.

\section{Conflicts of interest}

The authors have no conflicts of interest to declare.

\section{Author contributions}

All three authors contributed to the idea of writing this review. KA wrote the first draft. JPG and SP critiqued and added to the manuscript several times each to bring it to its current version. All authors approved the final version for publication.

\section{References}

1 National Institute for Health and Care Excellence. Chest pain of recent onset: assessment and diagnosis. NICE clinical guideline No 95. London: NICE, 2010.

2 Pryor DB, Shaw L, McCants CB et al. Value of the history and physical in identifying patients at increased risk for coronary artery disease. Ann Intern Med 1993;118:81-90.

3 National Institute for Health and Care Excellence. Chest pain. NICE pathway. Manchester: NICE, 2017. https://pathways.nice.org.uk/ pathways/chest-pain [Accessed 27 January 2017].

4 Montalescot G, Sechtem U, Achenbach S et al. 2013 ESC guidelines on the management of stable coronary artery disease: the Task Force on the management of stable coronary artery disease of the European Society of Cardiology. Eur Heart J 2013;34:2949-3003.

5 Genders TS, Steyerberg EW, Alkadhi $\mathrm{H}$ et al. A clinical prediction rule for the diagnosis of coronary artery disease: validation, updating, and extension. Eur Heart J 2011;32:1316-30.

6 Fihn SD, Gardin JM, Abrams J et al. 2012 ACCF/AHA/ACP/ AATS/PCNA/SCAI/STS guideline for the diagnosis and manage- 
ment of patients with stable ischemic heart disease: a report of the American College of Cardiology Foundation/American Heart Association task force on practice guidelines, and the American College of Physicians, American Association for Thoracic Surgery, Society for Cardiovascular Angiography and Interventions, and Society for Thoracic Surgeons. Circulation 2012;126:e354-471.

7 Cheng VY, Berman DS, Rozanski A et al. Performance of the traditional age, sex, and angina typicality-based approach for estimating pretest probability of angiographically significant coronary artery disease in patients undergoing coronary computed tomographic angiography: results from the multinational coronary CT angiography evaluation for clinical outcomes: an international multicenter registry (CONFIRM). Circulation 2011;124:2423-32.

8 Demir OM, Dobson P, Papamichael ND et al. Comparison of ESC and NICE guidelines for patients with suspected CAD: Evaluation of the pre-test probability risk scores. Clin Med 2015;15:234-8.

9 Bittencourt MS, Hulten E, Polonsky TS et al. European Society of Cardiology-Recommended Coronary Artery Disease Consortium pretest probability scores more accurately predict obstructive coronary disease and cardiovascular events than the Diamond and Forrester Score. Circulation 2016;134:201-11.

10 Douglas PS, Hoffmann U, Patel MR et al. Outcomes of anatomical versus functional testing for coronary artery disease. $N$ Engl J Med 2015;372:1291-300.
11 SCOT-HEART investigators. CT coronary angiography in patients with suspected angina due to coronary heart disease (SCOT-HEART): an open-label, parallel-group, multicentre trial. Lancet 2015;385:2383-91.

12 Abubakr M, Roobottom C, Morgan-Huges G. Appropriate use of down-stream invasive coronary angiography (ICA) following computed tomographic coronary angiography (CTCA) is still a work in progress. Heart 2016;102(Suppl 5):A1.

13 Fazel R, Krumholz HM, Wang Y et al. Exposure to low dose Ionizing radiation from medical imaging procedures. $\mathrm{N} \mathrm{Engl} \mathrm{J} \mathrm{Med}$ 2009;361:849-57.

14 Levsky JM, Spevack DM, Travin MI et al. Coronary computed tomography angiography versus radionuclide myocardial perfusion imaging in patients with chest pain admitted to telemetry: a randomized trial. Ann Intern Med 2015;163:174-83.

Address for correspondence: Dr Khaled Alfakih, University Hospital Lewisham, Lewisham High Street, London SE13 6LH, UK.

Email: khaled.alfakih@nhs.net

\title{
NGC
}

\section{New to systematic reviewing, or need to improve your critical appraisal skills?}

\author{
Our intensive 1-day courses will introduce you to the key \\ principles of critical appraisal and systematic reviewing, \\ and equip you with the basic skills to put your knowledge \\ into practice.
}

\section{$>$ Introduction to critical appraisal > Systematic reviews and meta-analysis in action}

Discount available if both courses are booked together.

\section{NGC Short courses}

\section{'Informative and practical'} Dr Rajeswari Ramaraj

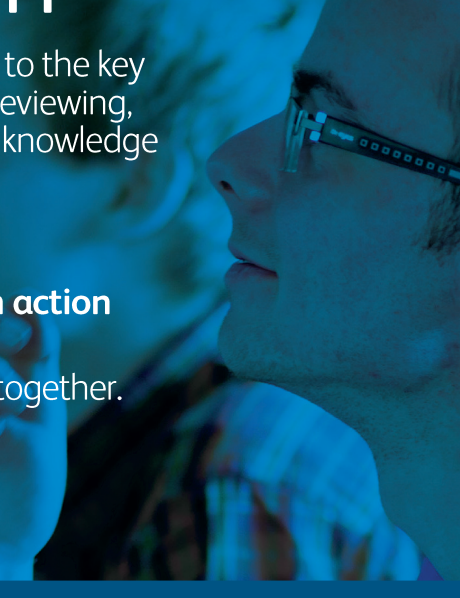

CPD credits

For course dates and more information visit: www.ngc.ac.uk/training-courses 